\title{
Correlation of circulating DNA (cDNA) alterations in several genes with established prognostic markers in a series of solid tumors treated with Multi Targeted Epigenetic therapy (MTET)
}

\author{
MA Nezami*, Steve Hager and Christian Klosowski \\ Pacific Medical Center of Hope, Pacific Medical Center of Hope, Sahel Oncology, Fresno, CA, USA
}

\begin{abstract}
In this article we review the dynamics of changes in the observed minor allele frequency fraction (MAF) as measured through circulating tumor DNA (ctDNA) of alterations in 8 target genes: NOTCH1, FGF2, MYC, RB1, ATM, MET, BRCA1, BRCA2, ATM, and VHL for which epigenetic dysregulation is identified in the literature to be most relevant to clinical response. We retrospectively examine a series of 54 oncology patients treated with multitargeted epigenetic therapy (MTET), a protocol used to treat solid tumors with epigenetically influenced driver genes. We examined the additional prognostic value of tracking such measurements of MAF with relation to their outcome, in the context of established survival markers including circulating tumor cells and PET imaging. In our cohort, observed levels of ctDNA changed rapidly marking it as a potential indicator for early molecular response. More than 50 percent reduction of ctDNA MAF and/or complete disappearance of CTC was used as major response after two weeks of the therapy. We concluded that molecular response identified in these 8 dysregulated genes, had independent prognostic value for outcome and could be used to generate further hypothesis in larger studies in solid tumors treated with this method. While previous research into the prognostic value of tracking ctDNA change has largely focused on specific cancer types or a specific gene, our study tracks several target genes over a variety of cancer types.
\end{abstract}

\section{Introduction}

Genetic and epigenetic dysregulation of genes involved in pathways such as the hypoxia-inducible pathway, the mTOR pathway, and the cMET-RAF-MEK-ERK pathway contribute to the progression of different types of cancers. Determination of disseminated tumour cells by genotyping of oncogenes and tumour suppressor genes seems not only to be a useful adjunct in follow up of carcinoma patients but provides also valuable additional individualized prognostic and predictive information in patients beyond the TNM system [1,2].

Research on tumour biology has provided conclusive evidence on the primary role of genetic alterations in the initiation and progression of cancer. However, the deregulation of epigenetic processes such as DNA methylation and alterations of "histone code" are equally important oncogenic factors per se. That said, recent trials have showed that epigenetic agents only exert favorable modifier effects when combined with chemotherapy, hormonal therapy, or other epigenetic agents. So far, epigenetic therapies, including HDAC inhibitors and demethylating agents, show considerable activity in hematological malignancies, but their value as a sole therapy or combined with targeted therapies, in the treatment of solid tumours remains much more uncertain. Due to the novel nature of their mechanism, it is important to reconsider the optimal patient selection, drug regimen, study design, and outcome measures when pursuing future trials in order to discover the full potential of this new therapeutic modality [3-5].

One crucial goal of patient treatment is to improve survival while maintaining the quality of life and avoiding needless toxic effects of an ineffective treatment, especially for patients without curative intent. The ability to evaluate therapeutic efficacy early in treatment is a key to achieving this goal.

The current gold standard for assessing tumor response and treatment efficacy is the radiographic imaging based on the Response Evaluation Criteria in Solid Tumors (RECIST) criteria. However, there are some limitations to its use: the evaluation occurs at least after 2 months of treatment, it can be assessed only in patients with measurable lesions, it has poor interobserver reproducibility, and the introduction of targeted therapies modifies the correlation between efficacy and tumor shrinkage [6-7]. Furthermore, serial radiographic imaging is expensive, time consuming, inconvenient, and contributes to an accumulation of ionizing radiation. Several attempts have been made to introduce biomarkers for response evaluation without strong success.

The role of circulating DNA in identification of early relapse and surrogate marker for therapy response in large B cell lymphoma has recently been investigated $[8,9]$. In another study, the presence of ctDNA had a higher positive predictive value than that of six tumor biomarkers

${ }^{*}$ Correspondence to: MA Nezami, MD, President, Pacific Medical Center of Hope, Pacific Medical Center of Hope, Sahel Oncology, Fresno, CA, USA, E-mail: amnezami@yahoo.com

Key words: multi targeted epigenetic therapy, circulating DNA

Received: April 01, 2019; Accepted: April 23, 2019; Published: April 25, 2019 
Nezami MA (2019) Correlation of circulating DNA (cDNA) alterations in several genes with established prognostic markers in a series of solid tumors treated with Multi Targeted Epigenetic therapy (MTET)

in current clinical use for non-small cell lung cancer suggesting plasma ctDNA could be effectively used to monitor NSCLC patients during treatment [10-12]. Unlike certain tumor protein biomarkers used to monitor disease progression that lack sensitivity and specificity plasma ctDNA concentrations have been shown to correlate well with tumor burden, although additional factors such as the type of tumor also factor into plasma ctDNA concentration [13,14]. Additionally, it has been demonstrated that plasma ctDNA may be an independent prognostic biomarker for NSCLC, where patients with higher plasma ctDNA were shown to have poor responses to treatment and worse survival rates than those with lower plasma ctDNA concentrations [15].

Other analysis has shown levels of mutations in specific genes identified in cfDNA correlating with outcome. One study found the identified plasma mutant KRAS levels strongly predicted response to an EGFR inhibitor in metastatic colorectal cancer patients [16]. In another study in breast cancer patients, the most commonly found genomic alterations were mutations in TP53 and PIK3CA, and amplification of EGFR and ERBB2. The presence and the frequency of mutant allele frequencies fraction (MAF) for both TP53 and PIK3CA mutations, correlated with individual's survival. Patients with PIK3CA mutant ctDNA were dichotomized into mutant ctDNA-high (ctDNAhigh) and ctDNA-low (ctDNAlow) groups based on the median. The ctDNAhigh patients exhibited significantly shorter recurrence-free survival and overall survival rates compared to those exhibited by the combined ctDNAlow patient and ctDNAfree patient group. It is suggested that this test can replace conventional tumor markers such as CEA and CA15-3, as these markers do not correlate well with the PFS or the tumor response. Prognostic significance of these alterations was not associated with the therapy which patient had received. It was also interesting to note that the size of the tumor did not correlate with the presence or quantity of the discovered PIK3CA alterations. That said tumor histology (and therefore behavior) was directly correlated to this. In this regard, there was a correlation with epigenetic state of the tumor at several genes, including GSTP1, RASSF1A, and RARbmethylated ctDNA $[17,18]$.

\section{Testing methods and materials}

Circulating tumor DNA (ctDNA) analysis was performed via Guardant360 as previously described $[19,20]$. Briefly, two $10 \mathrm{~mL}$ tubes of blood were collected in Streck Cell-Free DNA BCT (Streck, Inc.). Plasma was isolated via centrifuge, and cfDNA was extracted from the plasma and labeled with nonrandom oligonucleotide barcodes (IDT, Inc.). This cfDNA was then used to prepare sequence libraries which were enriched by hybrid capture (Agilent Technologies, Inc.) and sequence by paired-end synthesis (NextSeq 500 and/or HiSeq 2500, Illumina, Inc.). Variant analyses were performed using the locked Guardant 360 bioinformatics pipeline and reported unaltered by post hoc analyses. All decision thresholds were determined. Base call files generated by Illumina's RTA software (v2.12) were demultiplexed using bcl2fastq (v2.19) and processed with a custom pipeline for molecule barcode detection, sequencing adapter trimming, and base quality trimming.

Circulating Tumor Cell (CTC) analysis was performed via Biofocus (www.biofocus.de). Heparinized blood samples of patients were processed. In brief, CTCs from $30 \mathrm{ml}$ blood were enriched by filtration cytometry [30] using $20 \mu \mathrm{m}$ polyester filter meshes (Reichelt Chemietechnik, Heidelberg, Germany). RNA was extracted from cells retained on filter meshes with Trizol reagent. For proof of CTCs in these cell preparations, qRT-PCR for relative mRNA expression of a set of four genes (telomerase, ERBB2, c-KIT, EGFR) was performed. Assays were purchased (telomerase: Hs00972649, Applied Biosystems) or designed in-house taking care that fluorescence probes are spanning exon-boundaries [19]. Expression values in the enriched CTC preparation was normalized to the house-keeping gene GAPDH and compared to GAPDH-normalized expression values in mononuclear cells of the patients. Relative expression ratios of $>2.0$ (telomerase, c-KIT, ERBB2) or $>1.0$ (EGFR) in enriched CTC preparations were considered overexpressed and CTC-positive.

\section{Statistical analysis}

Sample size determination: We planned to assess the correlation value of $\mathrm{c}$ DNA on known factors for prediction of

progression-free survival (PFS) and overall survival (OS), recognized as surrogates for survival. For patients with metastatic cancer, there are four well-characterized surrogates: CTC, Imaging findings, metabolic activity in PET scan and angiogenic factors. Thus, our model will contain at most four prognostic factors.

A number of genes of interest, namely NOTCH1, FGF2, MYC, RB1, ATM, MET, BRCA1, BRCA2, ATM and, VHL, were selected based on previous reports of being highly influenced by epigenetic control. All patients seen at [insert your preferred name for your clinics in publications] who had received ctDNA analysis both before and after the initiation of MTET, who were found to have a mutation in at least one of the genes of interest, and had follow-up data with an orthogonal biomarker were included in this analysis.

In this case, in absence of other data, in particular of prespecified cutoff, it is not formally possible to determine the appropriate sample size. However, according to Ogundimu and colleagues, it is necessary to have at minimum of 10 events per variable of a model to develop a statistically stable prognostic model [19]. We therefore needed to include at least 40 patients for this study evaluating the predictive impact of early changes in cDNA on survival. Therefore, we attempted to exceed the required sample size and selected all patients with reported changes in their CDNA on the genes of interest to our study, namely NOTCH1, FGF2, MYC, RB1, ATM, MET, BRCA1, BRCA2, ATM and, VHL, for a total of 54 subjects. These genes are reported to be highly influenced by the epigenetic control.

There were 12 cases with NOTCH1 mutations, 20 cases with MYC mutations, 5 cases with RB1, 15 cases with BRCA1 or BRCA2 mutations, 26 cases with FGF2 mutations, 18 cases with MET mutations, 2 with VHL mutations and 2 cases with ATM mutations, for a total of 100 data points. All these subjects had post therapy evaluation with follow up testing (Table 1).

Significance Testing: Tests for statistical significance were completed using the Fisher Exact probability test calculator found at vassarstats.net/tab2x2.html. Significance level was set at $\alpha=0.05$.

Results: In the group of patients with NOTCH1 alterations, 9/12 had decreased allele frequency in their ctDNA post-therapy. 7 of these 9 cases showed similar response in other biomarkers defined by disappearance of CTC or marked reduction of the EpCAM markers. All individuals with improved VEGF had positive response in c DNA and CTC. There were 7 cases with measurable imaging response and/ or tumor marker reduction. There was statistically significant ( $\mathrm{p}$-value: 0.046) correlation on the response of the allele frequency of the observed NOTCH1 alteration with similar response in the angiogenesis biomarkers (Plasma VEGF) and presence of circulating tumor cells. 
Nezami MA (2019) Correlation of circulating DNA (cDNA) alterations in several genes with established prognostic markers in a series of solid tumors treated with Multi Targeted Epigenetic therapy (MTET)

Table 1. Demographics for the subset of cases

\begin{tabular}{|c|c|c|c|c|}
\hline $\begin{array}{l}\text { Demographics } \\
\text { Alteration }\end{array}$ & $\begin{array}{l}\text { Male/ Female } \\
\text { distribution }\end{array}$ & $\begin{array}{c}\text { Number of } \\
\text { subjects }\end{array}$ & Average age & $\begin{array}{c}\text { Ethnicity } \\
\text { Caucasian (C) } \\
\text { Asian (A) } \\
\text { Hispanic (H) }\end{array}$ \\
\hline NOTCH 1 & $3 / 9$ & 12 & 52 & $\begin{array}{c}\text { 7(C), } 2(\mathrm{~A}) \\
3(\mathrm{H})\end{array}$ \\
\hline С Myc & $6 / 14$ & 20 & 58 & $\begin{array}{c}14(\mathrm{C}), 1(\mathrm{~A}) \\
5(\mathrm{H})\end{array}$ \\
\hline FGF-2 & $5 / 20$ & 25 & 55 & $\begin{array}{c}18(\mathrm{C}) \\
3(\mathrm{~A}), 4(\mathrm{H})\end{array}$ \\
\hline
\end{tabular}

In the group of 20 cases with MYC alterations, 3 of them had NOTCH1 alteration as well. Many individuals with MYC alterations identified in cfDNA also had circulating tumor cells present before. Such correlation was observed along with the course of therapy with reduction of MAF and CTC. 16 of the 20 individuals with MYC alterations saw decreased AF after initiation of MTET. Of these 16, 12 cases demonstrated measurable response by imaging and/ or tumor markers, and 5 cases additionally showed reduced markers for angiogenesis post therapy. Of the four cases that did not show a significant change in AF after initiation of MTET, none showed biomarker or imaging response upon follow-up. There was a significant correlation (p-value: .014) between a decrease in AF of the observed MYC alteration and biomarker/imaging response on follow-up

In the group of 26 with FGF2 mutation identified in cfDNA, 22 saw allele frequency decrease therapy. 16 out of 26 cases had either c Myc or NOTCH1 alterations as well, 8 out of 18 cases with c Met alterations also shared NOTCH1 or Myc alteration, pointing to the common epigenetic driver of the genome instability. This represents a statistically significant (p-value: .0001) correlation on the response of the allele frequency of the observed FGFR2 alteration with the response of other biomarkers

Both cases with ATM alteration and all 5 individuals with RB-1 alteration had reduction of both ctDNA MAF as well as CTC markers for EpCAM. Response rate of individuals with these alterations were 100 percent. Imaging findings confirmed response in both ATM altered cases and $3 / 5$ of cases with RB- 1 alteration. In $2 / 5$ cases with $\mathrm{Rb}-1$ alteration, the CTC was measured and in both cases the response was consistent with the observed reduction in cfDNA with marked reduction of markers. In the group of patients with BRCA1/2 alterations, the angiogenesis biomarkers were only elevated in 4 cases, but all responded to the therapy. There was again marked correlation with CTC response and ctDNA response at molecular level. All cases with positive CTC response had positive response at their c DNA level [6]. Both cases with VHL alteration had major response (more than 50 percent reduction in MAF) at ctDNA level correlated one with CTC response and one with VEGF responses, both with evident positive imaging response.

\section{Limitations}

This study reports nonselective cases to avoid selection bias. Study however is limited in its stratification for other confounders, such as prior therapies. A lack of negative controls (positive response to therapy in all cases) made it impossible to perform Fisher Exact tests for RB-1, ATM, VHL, and BRCA2.

\section{Conclusion}

There were a number of significant correlations observed between early changes in cDNA allele fractions of specific genes, in response to the interim MTET therapy and the later observations of established prognostic markers, providing a meaningful companion test for this particular epigenetic therapy. We conclude that changes in the MAF of alterations observed on liquid biopsy may provide an early independent prognostic marker for tumors carrying epigenetically influenced targets and their alterations. We propose further investigation into prognostic value of changes in MAF for tumor response and molecular response in a larger sample. Further studies are indicated to explore the use of changing MAF of observed cDNA alterations as an early prognostic marker for the application of epigenetic therapies-based approach in these tumors.

\section{References}

1. Banumathy G, Cairns P (2010) Signaling pathways in renal cell carcinoma. Cancer Biol Ther 10: 658-664. [Crossref]

2. Austrup F, Uciechowski P, Eder C, Bockmann B, Suchy B, et al. (2000) Prognostic value of genomic alterations in minimal residual cancer cells purified from the blood of breast cancer patients. Br J Cancer 83: 1664-1673.

3. Juo YY, Gong XJ, Mishra A, Cui X, Baylin SB (2015) Epigenetic therapy for solid tumors: from bench science to clinical trials. Epigenomics 7: 215-235.

4. Clara Nervi, Elisabetta De Marinis, Giovanni Codacci-Pisanelli (2015) Epigenetic treatment of solid tumours: a review of clinical trials. Clin Epigenetics 7: 127.

5. Janet S, Graham, Stanley (2009) European Journal of Cancer, 45: 1129-1136

6. Sharma MR, Maitland ML, Ratain MJ (2012) RECIST: no longer the sharpest too in the oncology clinical trials toolbox---point. Cancer Res 72: 5145-5149. [Crossref]

7. Zhao B, Lee SM, Lee HJ, Tan Y, Qi J, et al. (2014) Variability in assessing treatment response: metastatic colorectal cancer as a paradigm. Clin Cancer Res 20: 3560.

8. Fanny Garlan, Pierre Laurent-Puig, David Sefrioui Nathalie, Siauve Audrey, Valerie Taly, et al., (2017) Early Evaluation of Circulating Tumor DNA as Marker of Therapeutic Efficacy in Metastatic Colorectal Cancer Patients (PLACOL Study). Clinical Cancer Research.

9. Kurtz DM, Scherer F, Jin MC, Soo J, Craig AFM, et al. (2018) Circulating tumor DNA measurements as early outcome predictors in diffuse large b-cell lymphoma. $J$ Clin Oncol 36: 2845-2853. [Crossref]

10. Nannan Guo, Feng Lou, Yongfu Ma, Jie Li, Bo Yang, et al. (2016) Circulating tumor DNA detection in lung cancer patients before and after surgery. Scientific Reports.

11. Sorensen (2014) Monitoring of epidermal growth factor receptor tyrosine kinase inhibitor-sensitizing and resistance mutations in the plasma DNA of patients with advanced non-small cell lung cancer during treatment with erlotinib. Cancer 120: 3896-3901

12. Murtaza M, Dawson SJ, Tsui DW, Gale D, Forshew T, et al. (2013) Non-invasive analysis of acquired resistance to cancer therapy by sequencing of plasma DNA. Nature 497 108-112. [Crossref]

13. Bast RC Jr, Xu FJ, Yu YH, Barnhill S, Zhang Z, et al. (1998) CA 125: the past and the future. Int J Biol Markers 13: 179-187. [Crossref]

14. Koprowski H, Herlyn M, Steplewski Z, Sears HF (1981) Specific antigen in serum of patients with colon carcinoma. Science 212: 53-55.

15. Tissot C (2015) Circulating free DNA concentration is an independent prognostic biomarker in lung cancer. European Respiratory Journal.

16. Karen-Lise Garm Spindler, Niels Pallisgaard, Ivan Vogelius (2012) Quantitative CellFree DNA, KRAS, and BRAF mutations in plasma with cetuximab and irinotecan from patients with metastatic colorectal cancer during treatment. Clin Cancer Res 18: 11771185 .

17. Atsushi Shimomura, Naomi Maruyama, Kenzo Shimazu, Seung Jin, Kim Shinzaburo et al. PIK3CA mutations in serum DNA are predictive of recurrence in primary breas cancer patients. Breast Cancer Research Treat.

18. Diana H. Liang, Joe E. Ensor, Zhe-bin Liu, Asmita Patel, Tejal A. Patel (2015) Cell-free DNA as a molecular tool for monitoring disease progression and response to therapy in breast cancer patients, Rodriguez. Breast Cancer Res Treat. 
Nezami MA (2019) Correlation of circulating DNA (cDNA) alterations in several genes with established prognostic markers in a series of solid tumors treated with Multi Targeted Epigenetic therapy (MTET)

19. Gundimu EO, Altman DG, Collins GS (2016) Adequate sample size for developing prediction models is not simply related to events per variable. J Clin Epidemiol 76: 175 .
20. Odegaard JI, Vincent JJ, Mortimer S, Vowles JV, Ulrich BC, et al. (2018) validation of a plasma-based comprehensive cancer genotyping assay utilizing orthogonal tissue- and plasma-based methodologies. Clin Cancer Res 24: 3539-3549. [Crossref]

Copyright: (C2019 Nezami MA. This is an open-access article distributed under the terms of the Creative Commons Attribution License, which permits unrestricted use, distribution, and reproduction in any medium, provided the original author and source are credited. 